\title{
SUBNICHO ESTRUCTURAL Y DENSIDAD POBLACIONAL DE CERION POLITUM MAISIANUM Y POLYMITA BROCHERI EN PASO DE LOS AZULES, MAISÍ, CUBA
}

\author{
Alexis Suárez Torres ${ }^{1}$ y Alejandro Fernández Velázquez ${ }^{2}$ \\ ${ }^{1}$ Est.01 La Española. MINAL. 35 No.2627 e/ 26 A y Final. Sta. Ma. del Rosario. CP 19330. \\ La Habana, Cuba. alexys4202@yahoo.com \\ ${ }^{2}$ Centro de Investigaciones y Servicios Ambientales y Tecnológicos. CISAT-CITMA-Holguín, Cuba. \\ ale@cisat.cu
}

\section{RESUMEN}

El hallazgo de individuos de una población de Cerion politum maisianum Pilsbry, 1902, cohabitando con Polymita brocheri (Gutiérrez , 1864), en una zona correspondiente a la localidad de Paso de los Azules, en Punta de Maisí, Guantánamo, Cuba, después de haberse realizado una prospección en el área, indica el estado en que se hallan estas especies. De acuerdo a las escalas propuestas y aplicadas, $P$. brocheri y $C$. politum maisianum resultaron Muy abundantes. Se da a conocer las preferencias entre éstas especies por sustratos diferentes, habiéndose encontrado la mayoría de ejemplares de $P$. brocheri a una altura $\geq 0.50 \mathrm{~m}$ y los de $C$. politum maisianum a una altura $<0.25 \mathrm{~m}$, condición que posibilita su coexistencia.

Palabras claves: Cerion politum maisianum, Polymita brocheri, sustrato, altura desde el suelo, densidad poblacional.

Title: Structural subniche and population density of Cerion politum maisianum and Polymita brocheri at Paso de los Azules, Maisí, Cuba.

\section{ABSTRACT}

The finding of individuals from the population of Cerion politum maisianum Pilsbry, 1902, cohabiting with individuals of Polymita brocheri (Gutiérrez, 1864) in one zone belonged to Paso de los Azules at Punta de Maisí, Guantánamo, after a research that was made in the area, it shows us the state that these species actually are. According to the proposal and applied scales $P$. brocheri and $C$. politum maisianum, both turned out Very abundant. The preferences on different substrates of these species are given too, finding the majority of the examples of $P$. brocheri at one height $\geq 0.50 \mathrm{~m}$ and $C$. politum maisianum examples at one height $<0.25 \mathrm{~m}$, condition that makes possible its coexistence.

Key words: Cerion politum maisianum, Polymita brocheri, substratum, height from the soil, populatiom density.

\section{INTRODUCCIÓN}

Las cinco etapas históricas de la malacología terrestre cubana descritas por Maceira et al. (2011) sintetizan el conocimiento taxonómico y biológico de la gran diversidad de especies de este grupo zoológico en la isla de Cuba, siendo las investigaciones ecológicas muy prolíficas a partir del año 1980, principalmente con los géneros Polymita Beck, Liguus Montfort, Zachrysia Pilsbry y Caracolus Montfort (Maceira et al., 2011).

Las especies del género Polymita son los moluscos terrestres cubanos mejor estudiados. Se han publicado algunos datos sobre la ecología de P. brocheri en Punta de Maisí (Maceira, 2000). 
La investigación en este trabajo fue centrada a nivel poblacional en Cerion politum maisianum y Polymita. Brocheri, especies que cohabitan en la misma región, con el fin de conocer algunos aspectos relacionados con el subnicho estructural y dilucidar si existen o no diferencias de abundancia entre ambas.

\section{OBJETIVOS}

- Investigar el uso del sustrato que realizan P. brocheri y C. politum maisianum y la altura desde el suelo en que se localizan en Paso de los Azules, Maisí, provincia Guantánamo.

- Determinar el estado de conservación de estas especies empleando el índice de la densidad poblacional en esta localidad.

\section{MATERIALES Y MÉTODOS}

El área de trabajo se halla en los $20^{\circ} 14^{\prime} \mathrm{N}$ y $74^{\circ} 08^{\prime} \mathrm{W}$, aproximadamente a dos kilómetros al Suroeste del faro de Punta de Maisí, en la provincia de Guantánamo, localidad conocida como Paso de los Azules. Aquí fueron hallados cohabitando ejemplares de las especies C. politum maisianum y $P$. brocheri a una distancia aproximada de 210 metros de la línea de marea, en el matorral xeromorfo costero (según los criterios de clasificación de Capote y Berazaín, 1984).

El muestreo se realizó los días 19 y 20 de abril de 2010, entre las 7:00 a.m y la 1:00 p.m., en un transepto de 200 metros de largo por un (1) metro de ancho, revisando a nivel del suelo, la hojarasca, ramas, troncos, rocas y las plantas hasta 1.50 metros de altura desde el suelo. Ambas especies están establecidas a la orilla de un sendero que corre de Este a Oeste, extendiéndose perpendicularmente a la costa. La densidad poblacional fue estimada en 22 parcelas de $1 \mathrm{~m} \times 1 \mathrm{~m}$ marcadas al azar.

Los valores de abundancia para P. brocheri y C. politum maisianum dados en ind $/ \mathrm{m}^{2}$, fueron ponderados a valores absolutos con fines de clasificación en categorías de abundancia según la escala utilizada por Maceira et al. (2005): Muy rara, uno (1) a tres (3) ind $/ \mathrm{m}^{2}$; Rara, tres (3) a $18 \mathrm{ind} / \mathrm{m}^{2}$; Escasa, 18 a $80 \mathrm{ind} / \mathrm{m}^{2}$; Común, 80 a $350 \mathrm{ind} / \mathrm{m}^{2}$; Abundante, 350 a 1,500 ind $/ \mathrm{m}^{2} \mathrm{y}$ Muy abundante $>1,500 \mathrm{ind} / \mathrm{m}^{2}$. Por ejemplo, $0.25 \mathrm{ind} / \mathrm{m}^{2}$ equivale a 250 individuos en $1000 \mathrm{~m}^{2}$; $0.008 \mathrm{ind} / \mathrm{m}^{2}$ equivale a ocho (8) individuos en $1000 \mathrm{~m}^{2}$.

Se siguió la definición de Silva y Berovides (1982) y de Berovides et al. (1988) referente a los estados y dimensiones de las subdivisiones del nicho estructural. La altura desde el suelo a la que se ubican los moluscos sobre las plantas se midió desde el suelo propiamente dicho hasta la parte de la concha más cercana al mismo, para lo cual se utilizó una cinta métrica.

Los datos individuales de alturas de los moluscos fueron agrupados en las siguientes clases: $0<0.25 \mathrm{~m} ; 0.251 \mathrm{~m}<0.50 \mathrm{~m} ; \geq 0.510 \mathrm{~m}$ para ambas especies. Se tomaron datos del tipo de sustrato en que se encontraban todos los especímenes observados, identificándolos con las siguientes variables: vegetación (V), el que a su vez se subdividió en vegetación viva (V.V) y vegetación muerta (V.M), carso expuesto (C), y sobre, entre y debajo de la hojarasca (H).

La descripción y prueba estadística para la altura desde el suelo de C. politum maisianum y $P$. brocheri fue realizada a través de una Tabla de Contingencia F x C $(2 \times 2)$ para determinar si existe o no asociación de las especies de moluscos con dos clases de altura: clase $1(<0.50$ $\mathrm{m})$ y clase $2(\geq 0.50 \mathrm{~m})$. Como altura media se seleccionó para el análisis $0.50 \mathrm{~m}$ por ser la longitud vertical en que se solapan ambas especies. El procesamiento estadístico de los datos fue 
realizado mediante el programa para Window Statistica 6.1, StatSoft. Inc. 1984-2003 en http:// www.statsoft.com/.

En condiciones de campo fue identificada fácilmente $P$. brocheri. En el caso de $C$. politum maisianum aplicamos el trabajo de gabinete, utilizando las conchas vacías recolectadas, las cuales fueron comparadas conquiológicamente con ejemplares depositados en colecciones del Instituto de Ecología y Sistemática y del Museo Nacional de Historia Natural de Cuba (MNHNCu), ambos en La Habana además de haberse consultado la literatura especializada donde se describe originalmente la especie (Pilsbry, 1902).

\section{RESULTADOS Y DISCUSIÓN}

Sustrato. En el área pre-establecida en punta de Maisí correspondiente al matorral xeromorfo costero se contó un total de 80 ejemplares de $C$. politum maisianum; cuatro (4) especímenes se observaron sobre el suelo, mientras que el resto se encontraron sobre la vegetación. En el caso de $P$. brocheri, los 77 individuos observados estaban sobre ramas. No se hallaron ejemplares en actividad en ninguno de los dos casos, hallándose el 100\% de los ejemplares de ambas especies en hibernación o sopor invernal, como respuesta al período de sequía.

Un alto porcentaje de los especímenes de C. politum maisianum (95\%) se observó sobre partes muertas de la vegetación (Fig.1) y un 5\% sobre carso. Este resultado constituye la primera notificación donde se da a conocer que Cerion utiliza como sustrato plantas muertas. Esto no contradice que especies del género Cerion Röding, 1798 ocupen diferentes sustratos. Existen trabajos donde se registra el uso de diferentes sustratos por Cerion, se ha referido para $C$. paucicostatum paucicostatum Torre, 1929 el uso de ramas, troncos de arbustos y en el carso (Maceira, 2000). Un estudio de historia natural realizado con Cerion alberti Clench y Aguayo, 1949, en Antilla, provincia Holguín, demostró que esta especie se encuentra restringida al suelo, sobre emergencia cársicas y sustrato rocoso arenoso (Suárez et al., en prensa).

Otros autores mencionan que Cerion tiene preferencias por las costas de arrecifes y por las playas de arena, viviendo sobre diversas especies de plantas, pero con marcada preferencia por uveros, jaguas, palmas, etc. En muy raras ocasiones se encuentran sobre mangles. Son de actividad nocturna y de día se encuentran adheridos (o estivando) a los troncos y ramas de los arbustos, a pleno sol, rara vez están debajo de piedras. Algunas formas viven en vegetación corta, próximas a lagunas salobres (Jaume, 1975); "viven en zonas secas y arenosas, contiguas a playas y seborucos, en un ambiente con marcada influencia marina, por lo general a no más de 800 metros de la línea de mareas" (Espinosa y Ortea, 2009); "ocupan un hábitat precario, particularmente entre las uvas caletas y otras plantas asociadas" (Clench y Aguayo, 1952); o viven "en la tierra debajo de la vegetación en áreas abiertas, no enterrados, en la hierba y debajo de las rocas" (Galler y Gould, 1979).

Los moluscos terrestres pulmonados pueden ocupar diferentes subnichos estructurales: Coryda alauda Férrussac, 1821, muestra preferencia por las ramas; Caracolus sagemon Beck, 1837, prefiere los troncos; P. picta Born, 1780, sobre hojas (Bidart y Espinosa, 1989); Hemitrochus lucipeta Poey, 1854, ha presentado preferencias por hojas; Polymita muscarum Lea, 1834, presenta preferencia por ramas y Liguus fasciatus Müller, 1774, por troncos y ramas (Bidart et al., 1992). Cambios temporales en el uso del subnicho estructural también se han reportado para L. fasciatus (Fernández, 2005).

Los resultados obtenidos en $P$. brocheri coinciden con otros autores (Valdés et al., 1986; Berovides, 1987; Bidart et al, 1989; Bidart et al., 1992; Bidart, 1997; Fernández, 1990, 2005; 
Fernández et al., 2000), quienes observaron varias especies de moluscos arborícolas cubanos sobre ramas y troncos durante la hibernación.

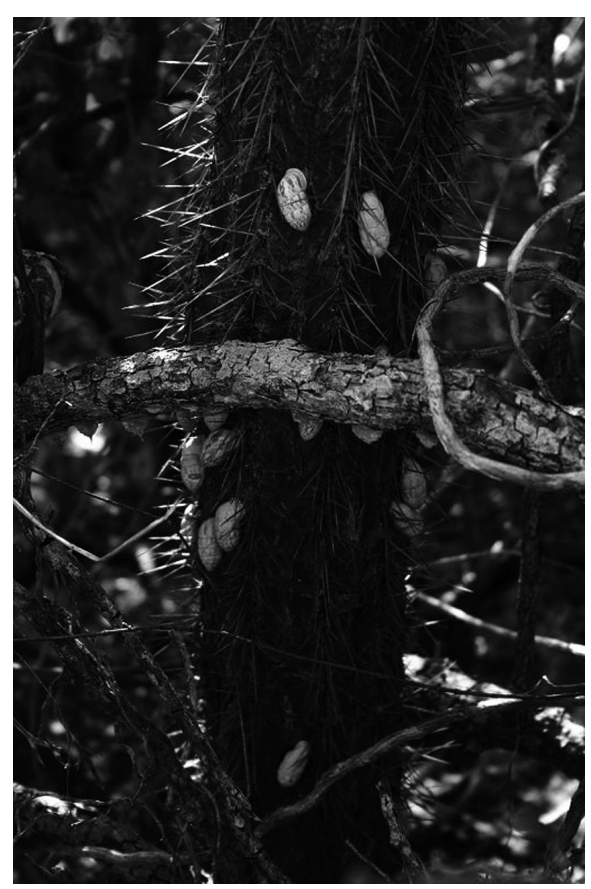

Figura1. Cerion politum maisianum sobre cactácea en descomposición.

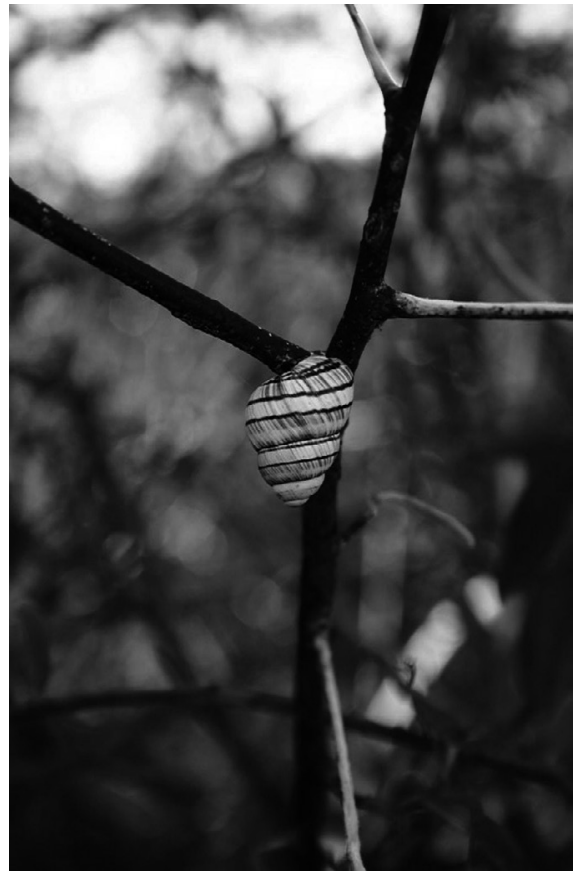

Figura 2. Polymita brocheri en rama de planta viva.

Altura desde el suelo. E1 80\% de ejemplares de C. politum maisianum fueron encontrados a una altura menor que los de $P$. brocheri. La mayor altura que presentó $C$. politum maisianum desde el suelo correspondió al $71.4 \%$ de los individuos $(0.50 \mathrm{~m})$ y sólo pocos individuos solapan su distribución vertical dentro de la planta; un $17.5 \%$ de $C$. politum maisianum se halla en la clase de $0.25<0.50 \mathrm{~m}$; estos resultados evidenciaron segregación vertical de ambas especies dentro del mismo hábitat.

El análisis de los datos anteriores, mediante una Tabla de Contingencia $(\mathrm{FxC}) \mathrm{X}^{2}(2 \times 2)\left(\mathrm{X}^{2}=\right.$ $80.61 \mathrm{df}=1 ; \mathrm{p}<0,0001)$, evidenció fuerte asociación de ambas especies en la diferenciación con las clase de altura desde el suelo de los moluscos (Tabla 1), por lo que se demuestra estadísticamente la segregación de este carácter relacionado con el subnicho estructural.

Densidad poblacional. En abril del 2010, P. brocheri tuvo una densidad poblacional de 3.5 ind $/ \mathrm{m}^{2}$, lo que ubica a la población en la categoría de Muy abundante. Estos valores resultaron superiores a los estimados en agosto de 1998 en Punta de Maisí, para cuya localidad se reportaron $0.12 \mathrm{ind} / \mathrm{m}^{2}$ (Maceira, 2000).

C. politum maisianum tuvo una densidad de 3.6 ind $/ \mathrm{m}^{2}$, valor muy semejante al de $P$. brocheri $\left(3.5 \mathrm{ind} / \mathrm{m}^{2}\right)$ por lo que ambas especies podrían no estar afectadas demográficamente por competencia ni por la actividad humana. Sus subnichos estructurales están bien diferenciados, hecho que posibilita la coexistencia, con gran similitud de abundancia poblacional. 
Tabla. 1. Descripción y prueba estadística de la altura desde el suelo de $C$. politum maisianum y $P$. brocheri (clase $1,<0.50 \mathrm{~m}$; clase $2, \geq 0.50 \mathrm{~m}$ ) en matorral xeromorfo costero en Paso de los Azules, abril 2010 .

\begin{tabular}{|l|l|l|l|}
\hline & Cerion politum maisianum & Polymita brocheri & Total \\
\hline Frecuencia, clase 1 & 78 & 22 & 100 \\
\hline Porcentaje & 49.68 & 14.01 & 63.69 \\
\hline Frecuencia, clase 2 & 2 & 55 & 57 \\
\hline Porcentaje & 1.27 & 35.03 & 36.31 \\
\hline Total por especie & 80 & 77 & 157 \\
\hline Porcentaje & 50.96 & 49.04 & 100 \\
\hline Chi-cuadrado $(\mathrm{df}=1)$ & $80.61 ; \mathrm{P}=0.0000$ & \\
\hline Corrección de Yates & $77.66 ; \mathrm{P}=0.0000$ & \\
\hline
\end{tabular}

Las especies de C. politum maisianum y $C$. paucicostatum paucicostatum no cohabitan en La misma localidad, aunque se hallan geográficamente muy cercanas. Para esta última se determinó una densidad poblacional de $0.12 \mathrm{ind} / \mathrm{m}^{2}$ (Maceira, 2000), restringiéndose a la vegetación de costa rocosa y complejo de vegetación de costa arenosa encontrándose en ramas, troncos de arbustos y en el carso.

La población de C. politum maisianum se halla más alejada de la costa, en matorral xeromorfo costero y según las categorías de abundancia referidas por Maceira et al. (2005) se clasifica dentro de la categoría de Muy abundante, a diferencia de $C$. paucicostatum paucicostatum, el que habita en una zona más cercana a la costa y posee una menor densidad. Varias pueden ser las causas que determinan estas diferencias entre ambas especies, la franja litoral que ocupa la vegetación de costa rocosa y el complejo de vegetación de costa arenosa es más estrecha que el espacio ocupado por el matorral xeromorfo costero. La ausencia de depredadores y los hábitats sin perturbaciones naturales o antrópicas también podrían repercutir en la salud de la población. Por consiguiente, C. politum maisianum, según la categoría anteriormente mencionada, se cataloga también de Muy abundante. Este criterio de clasificación de abundancia ubica claramente a $C$. politum maisianum como especie en buen estado de conservación.

Nuevos estudios en los géneros Polymita y Cerion, ayudarían al conocimiento del estado actual en que se hallan estas especies, sus areas de distribución, los cambios de abundancia espacio temporales, así como dilucidar cambios temporales del subnicho estructural.

\section{AGRADECIMIENTOS}

A Guillermo Ponce de León, Alexis Morales, Alexis Morales (hijo) y a Antonio Blet, sin cuya ayuda no hubiese sido posible este trabajo. A los Drs. David Maceira y José Espinosa, por la revisión crítica del documento y sugerencias oportunas. A Iriel Cobreiro, por su apoyo sin igual. A Tropas Guardafronteras de Baracoa y Punta de Maisí, Guantánamo, en especial al Capitán Yoanni Gámez.

\section{LITERATURA CITADA}

Berovides, V. 1987. Genética ecológica de Polymita picta roseolimbata Torre 1950, en la región de Maisí. Facultad de Biología. Universidad de La Habana. Resumen Tesis de Doctor en Ciencias Biológicas. 40 pp. 
Berovides, V., J. A. Genaro y C. Sánchez. 1988. Nuevas consideraciones acerca del nicho ecológico. Ciencias Biológicas, 19-20: 3-8.

Berovides, V. y M. A. Alfonso. 1995. Biología evolutiva. Editorial Pueblo y Educación. Ciudad de la Habana. 407 pp.

Bidart, L. 1997. Ecología de Polymita muscarum Lea 1834 (Gastrópoda: Xanthonychidae) en la provincia Holguín. Tesis en Opción al Título de Master en Ciencias de Ecología y Sistemática Aplicada. I. E. S. Ministerio de Ciencia, Tecnología y medio Ambiente, Ciudad de la Habana. 50 pp.

Bidart, L. y J. Espinosa. 1989. Aspectos del nicho ecológico de Polymita picta nigrolimbata, Caracolus sagemon rostrata y Coryda alauda strobilus. Ciencias Biológicas, 21-22: 130135.

Bidart, L., J. Espinosa y A. Pérez. 1989. Dinámica poblacional de Polymita picta nigrolimbata. Poeyana, 381. 16 pp.

Bidart, L., M. Osorio, E. J. Reinaldo, F. Milera, y C. Iglesias. 1992. Nicho ecológico de Polymita muscarum, Liguus fasciatus achatinus y Hemitrochus lucipeta. Ciencias Biológicas, 25: 9-19.

Capote R. y R. Berazaín. 1984. Clasificación de las formaciones vegetales de Cuba. Revista del Jardín Botánico Nacional, 5(2): 27-75.

Clench. W. J. y C.G. Aguayo, 1949. Algunas especies de Ceriónidos de la costa Norte de Oriente, Cuba. Museo Felipe Poey, Universidad de La Habana; Torreia, 14: 1-10.

Clench.W. J y C. G. Aguayo. 1952. The Scalarinum Species Complex (Umbonis) in the genus Cerion. Occ. Paper on Mollusks Museum of Comparative Zoology 1(17): 413-440.

Espinosa, J. y J. Ortea. 1999. Catálogo de moluscos terrestres del archipiélago cubano. Avicennia (Suplemento 2): 1-137.

Espinosa, J. y J. Ortea. 2009. Moluscos terrestres de Cuba. Finlandia. 191 pp.

Fernández, A. 1990. Ecología de Polymita muscarum (Gastrópoda: Fruticicollidae) en la provincia Holguín. Revista Biología, IV (1): 1-13.

Fernández, A. 2005. Plant species and strata selected by Liguus fasciatus achatinus Clench, 1934 (Mollusca: Orthalicidae) in semideciduous forest in El Yayal, Holguín province, Cuba. Of Sea and Shore 27 (2): 89-93.

Fernández, A., J. A. La O, C. Peña, P. González y S. Monteagudo. 2000. Ecological niche of Polymita muscarum (Lea) and other land snails in Pesquero Nuevo Beach's Lagoon, R. Freyre. Holguin . Cuba. Of Sea and Shore, 23(3): 125-130.

Galler, L. y S. J. Gould. 1979. The morphology of a "hibrid zone" in Cerion: variation, clines, and an ontogenetic relationship between two "species" in Cuba. Evolution 33(2): 714-727.

Gould, S. J. y D. S. Woodruff. 1986. Evolution and systematics of Cerion (Mollusca: Pulmonata) on New Providence Island: A radical revision. Bulletin of the American Museum of Natural History, 182 (4): 389-490. 
Gutiérrez, 1864. Polymita brocheri. p. 237. En: Louis Pfeiffer, 1860-1866. Novitates Conchologicae. Serie prima. Mollusca extramarina. Description et figures de conquilles extramarines nouvelles ou peu connues. Tomo II. Avec 36 planches coloriees.

Jaume, M. L. 1975. Catalogo de os moluscos terrestres cubanos del genero Cerion. (MoluscaPulmonata-Cerriidae). Con una biblografia general. Catalogo de la fauna Cubana. XXXVII No 51 .

Maceira, D. 2000. Malacocenosis del matorral xeromorfo en Punta de Maisí, Cuba. Biodiversidad de Cuba Oriental, Volumen IV: 58-61.

Maceira, D., B. Reyes-Tur, A. Fernández y B. Laurazón. 2005. Estado de las poblaciones de las especies del género Polymita Beck, 1837. En Maceira, D. y B. Reyes Tur. Eds. BIOECOWWF, Santiago de Cuba. 53 pp., 42 anexos, 10 figs., 7 tablas.

Maceira, D., J. Espinosa y A.M. Pérez. 2011. Historia de la malacología terrestre cubana 18392010. Gaia, Biodiversidad, medioambiente y sociedad (12): 1-48.

Pilsbry, H. A. 1902. Manual of Conchology (2): 218.

Quensen J.F. y D. S. Woodruff 1997. Associations between shell morphology and land crab predation in the land snail Cerion. Journal Ecology 11: 464-471.

Silva, A. y V. Berovides.1982. Acerca del concepto de nicho ecológico. Ciencias Biológicas, 8: 95-103.

Suárez, T., A. Fernández e I. Hernández. (En prensa). Nuevos datos sobre la distribución, abundancia y el hábitat de Cerion alberti (Mollusca: Cerionidae), en Antilla, Holguín, Cuba. Revista electrónica Ecovida. Vol. 3, No1. ISSN: 2076-281.

Valdés, G., V. Berovides y J. F. Milera. 1986. Ecología de Polymita picta roseolimbata Torre, en la región de Maisí, Cuba. Ciencias Biológicas, 15: 77- 93.

[Recibido: 11 de agosto, 2011. Aceptado para publicación: 18 de mayo, 2012] 